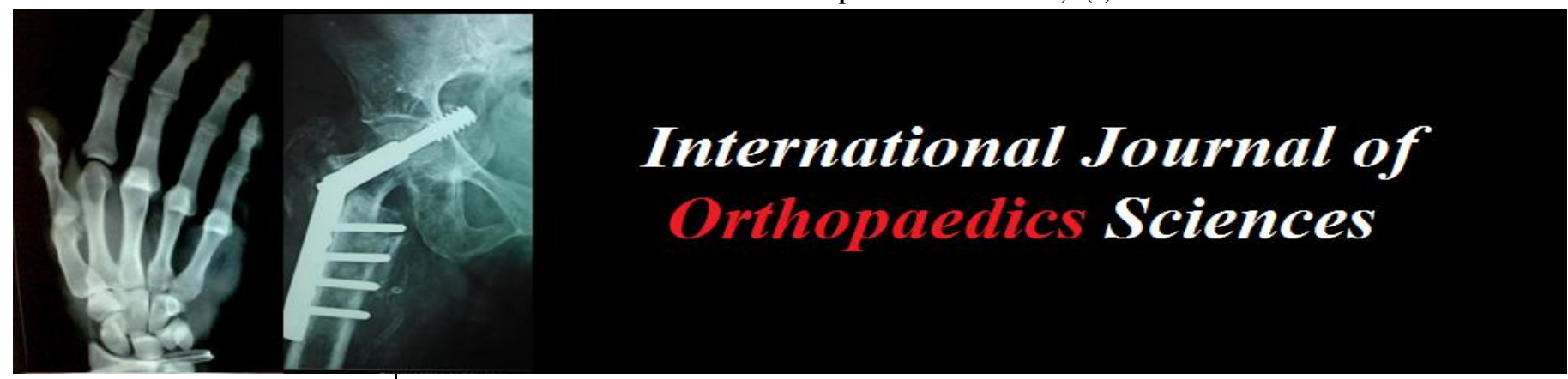

E-ISSN: 2395-1958

P-ISSN: 2706-6630

IJOS 2020; 6(2): 823-826

(C) 2020 IJOS

www.orthopaper.com

Received: 28-02-2020

Accepted: 30-03-2020

Gagandeep Mahi

Department of Orthopaedics,

GMCH, Aurangabad,

Maharashtra, India

Lokesh Kumar Yogi

Department of Orthopaedics,

GMCH, Aurangabad,

Maharashtra, India

Vijay Chandrakant Shinde Department of Orthopaedics, GMCH, Aurangabad,

Maharashtra, India

\section{Short term complications following uncemented hip hemiarthroplasty}

\author{
Gagandeep Mahi, Lokesh Kumar Yogi and Vijay Chandrakant Shinde
}

DOI: https://doi.org/10.22271/ortho.2020.v6.i2m.2145

Abstract

Introduction \& Objectives: Hemiarthroplasty is one of the most commonly done procedures and a recommended treatment for intra-capsular displaced fractures of femoral neck in elderly population ( $>65$ years). This study is aimed to evaluate the early complications in such patients following uncemented hemiarthroplasty using unipolar (Austin Moore Prosthesis) and bipolar implants.

Method: A series of 150 consecutive patients (between October 2017 and September 2018) treated with uncemented unipolar and bipolar hemiarthroplasty with at least one year follow-up were evaluated. Prospective data collection was attained from hospital and OPD visits viz. patient demographics, intraoperative iatrogenic fractures, deaths, deep infection, dislocation, peri-prosthetic fractures, implant failure and implant related complications.

Results: Average age of patients was 72 years (Range 65-101 years). 60\% patients were females. Intraoperative iatrogenic fractures occurred in 16 patients $(10.6 \%)$. There were two intra-operative deaths. Over all one year mortality was $10 \%$. There were 12 early deep wound infections (8\%). 6 dislocations were reported. There were 5 peri-prosthetic fractures. 2 patients required conversion to excision arthroplasty and 1 patient had implant breakage.

Keywords: Hip, fracture, uncemented, hemiarthroplasty

\section{Introduction}

In elderly hip fractures pose a major public health problem ${ }^{[1]}$. These fractures form around one fourth of all fractures in patients aged 75 years and over ${ }^{[2]}$. Femoral neck fractures occur most frequently in elderly female patients following fall from standing height and may be associated with osteoporosis ${ }^{[3]}$.

Femoral neck fractures in elderly individuals are commonly classified according to the Garden classification. Garden I and II (Un-displaced fractures) are usually managed with hip preservation, and Garden III and IV (Displaced fractures) are generally managed with arthroplasty (Hemi or Total) in elderly patients ${ }^{[4]}$.

Many surgical operations are available for displaced type femoral neck fractures with regard to acetabular changes, functional activities and age of patients ${ }^{[5]}$.

Cemented hemi or total arthroplasty are used more frequently but the possible effects of cement on the cardiopulmonary system and the greater technical challenge to revision of cemented hemi or total arthroplasty has led surgeons to prefer uncemented hemi or total arthroplasty ${ }^{[6]}$. Hemiarthroplasty is the more often surgical option currently used for the treatment of displaced intracapsular femoral neck fractures in elderly patients to restore their mobility and prevent of complications related to decreased ambulation following fracture ${ }^{[7]}$

The aim of this study is to ascertain the prevalence of various complications (e.g., intraoperative iatrogenic fractures, deaths, deep infection, dislocation, peri-prosthetic fractures, implant failure and implant related complications) during or after surgery.

\section{Methods}

This study done in a prospective manner with 12 months follow up of 150 patients with displaced femoral neck fractures treated with uncemented hemiarthroplasty through posterior approach at our hospital between October 2017 and September 2018.
Lokesh Kumar Yogi

Department of Orthopaedics,

GMCH, Aurangabad,

Maharashtra, India 
With regard to the operative technique, all procedures were performed with the patients under spinal or epidural anaesthesia and the patient positioned in lateral decubitus. Posterior approach (Moore's or Southern approach) was used: the femoral head and neck are removed; the femoral canal is prepared; unipolar (Austin Moore Prosthesis) and bipolar uncemented implants were used. Capsule and short external rotators were sutured back in layers. Mechanical and medical prophylaxis for DVT was used during the pre operative, immediate postoperative period, with active and passive physiotherapy and early mobilization for the patient.

Average duration of operation (in minutes) was $74.4( \pm 8.4)$ and $5.7( \pm 2.7)$ days was average post-operative length of stay. Post-operative $X$ rays were taken in all stable patients after shifting them from post-operative recovery room. Routine follow-up were performed with AP pelvis X-rays at 1 month, 3 months, 6 months, 9 months and at 12 months.

Complications were noted during operative, immediate postoperative and till one year follow-up period.

\section{Results}

Complete data (Table 1) were available for 135 of 150 patients $(90 \%)$, partial data for $15(10 \%)$. Average age of the patients was 72(7.3) years (Range 65-101 years). 90 patients $(60 \%)$ were females. Intra-operative iatrogenic fractures occurred in 16 patients (Figure 1). There were two intraoperative deaths and 13 patients were deaths in post operative follow up duration. Overall mortality was $10 \%$. There were 12 early deep wound infections. 6 dislocations were reported (Figure 2), 4 of these were treated with closed reduction and 2 required repeat procedures with one size larger head. There were 5 peri-prosthetic fractures. 1 patient had implant breakage (Figure 3).

Table 1: Frequency of medical and hip complications, n (\%)

\begin{tabular}{|c|c|c|}
\hline Complication & $\begin{array}{c}\text { Number of } \\
\text { patients }\end{array}$ & $\begin{array}{c}\text { Percentage } \\
\text { (n) }\end{array}$ \\
\hline Intra-operative iatrogenic fractures & 16 & $10.6 \%$ \\
\hline Death & 15 & $10 \%$ \\
\hline Deep wound infections & 12 & $8 \%$ \\
\hline Dislocation & 6 & $4 \%$ \\
\hline Peri-prosthetic fractures & 5 & $3.3 \%$ \\
\hline Implant breakage & 1 & $0.6 \%$ \\
\hline
\end{tabular}

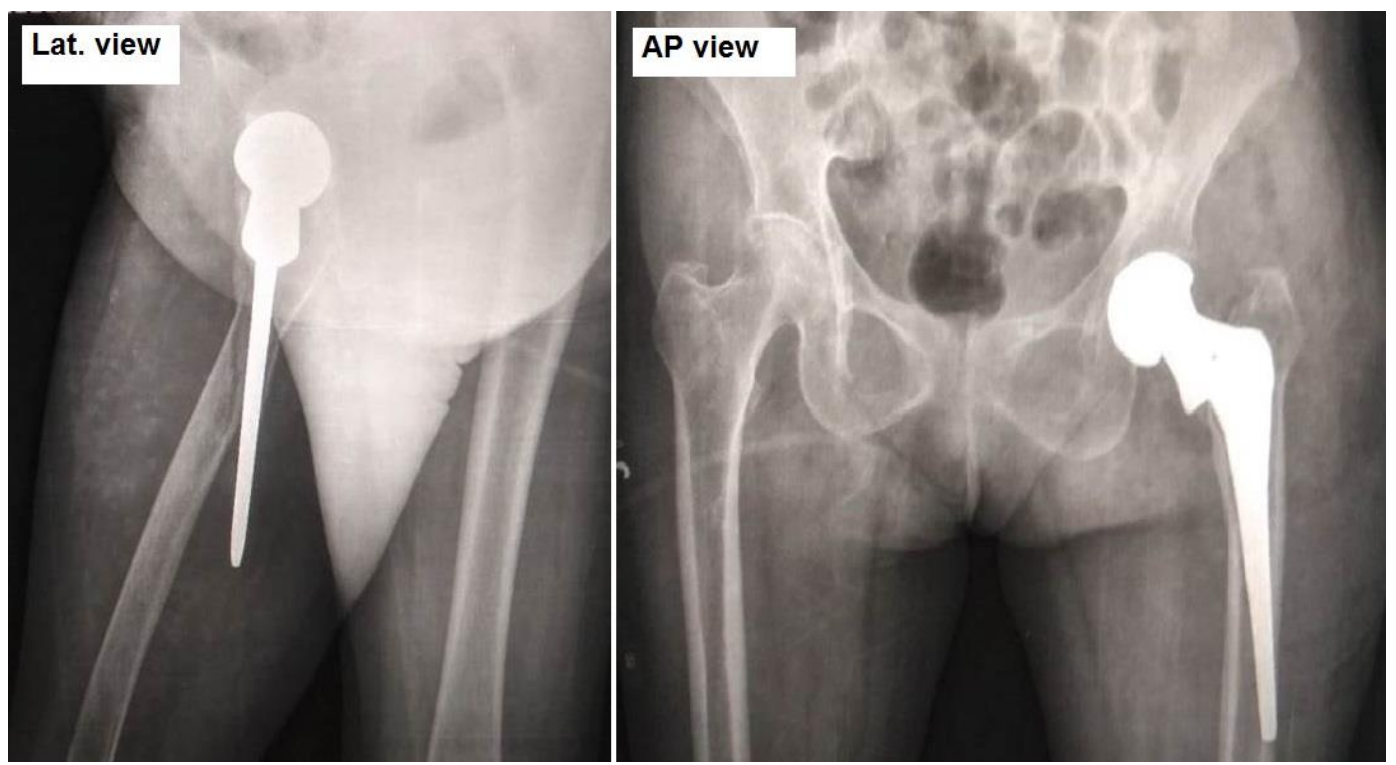

Fig 1: Intra-operative iatrogenic fractures in $\mathrm{AP}$ and Lateral view

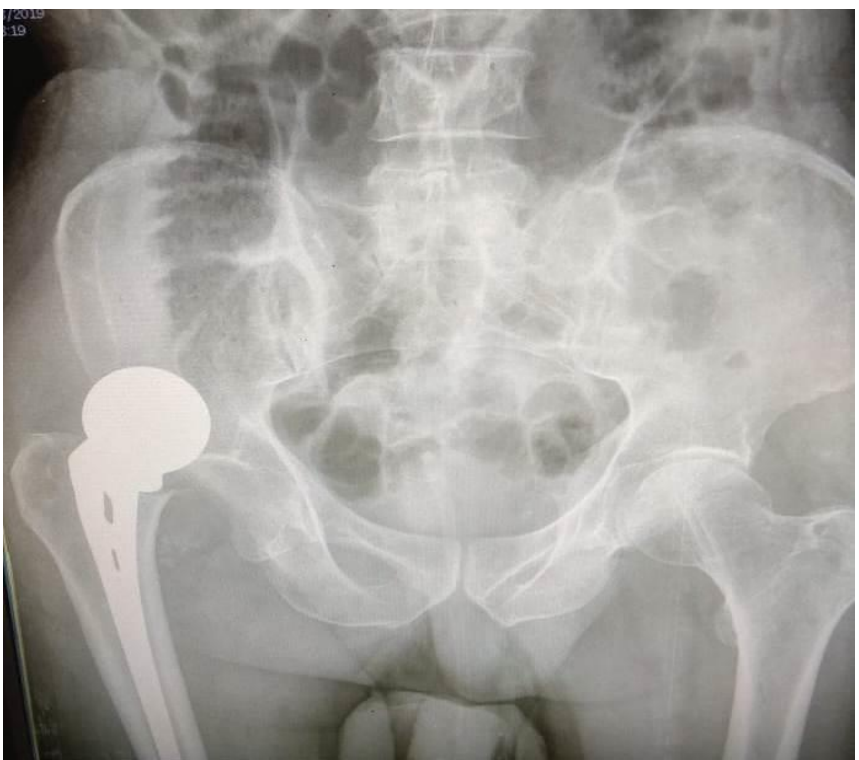

Fig 2: Post-operative prosthesis dislocation during follow up

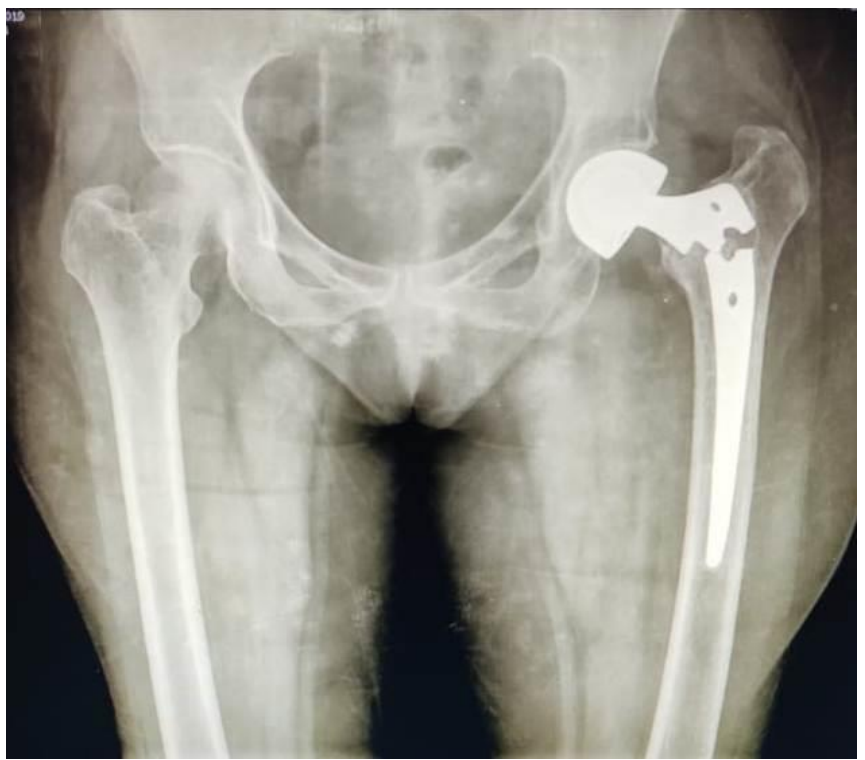

Fig 3: Post-operative implant breakage during follow up 


\section{Discussion}

A high number of elderly patients who have a femoral neck fracture have significant comorbidities as they are not very physically active. Hemi arthroplasty is found to be associated with less hip complications than total arthroplsty in intracapsular displaced neck fracture patients ${ }^{[8]}$. The most common modes of hemiarthroplasty complications in our study were intra-operative iatrogenic fractures, dislocation, death and deep infection. These complications are well recognised in the literature, especially when using uncemented hemiarthroplasty $[9,10]$. Early surgical complications after hemiarthroplasty may be the origin of cascades leading to general complications and increased mortality ${ }^{[3]}$.

In this study, intra-operative iatrogenic fractures occurred in $10.6 \%$ patients (14 patients were having greater trochanteric fractures and 2 had femoral shaft fractures), these patients managed with tension band wiring with k wires (Figure 4) and LCDCP plate with cerclage wiring respectively. Choon Chiet Hong et al. reported intra-operative iatrogenic fractures in $10.3 \%$. We found almost the same rate of complications in our patients ${ }^{[11]}$.

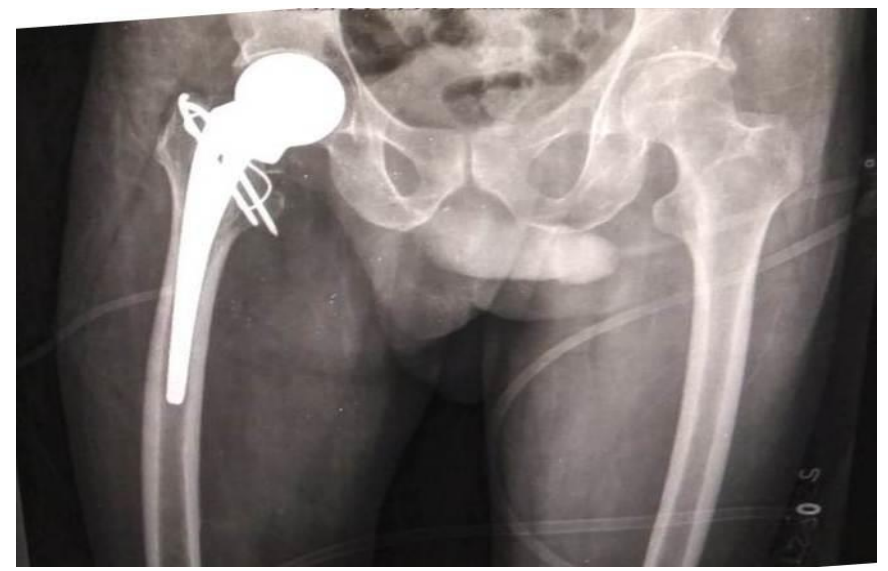

Fig 4: Greater trochanteric fracture managed with tension band wiring with $\mathrm{k}$ wires

The incidence of one year mortality was found $10 \%$ in this study that is nearly similar to Sadegh Saberi et al. study $8 \%$ one year mortality but this is less as compared to D'Angelo et al. and Eiskjar et al. studies, who reported $25 \%$ and $28 \%$ one year mortality respectively ${ }^{[3,12,13]}$. Deep infection was found in $8 \%$ of our patients and similar result was found in Chaplin VK et al. study ${ }^{[14]}$.

2 patients presenting with deep infection subsequently required conversion to excision arthroplasty procedure. The incidence of prosthesis dislocation in this study was found $4 \%$ as similar to that reported in Sadegh Saberi et al. and Chaplin $V K$ et al. studies $4 \%$ and $3 \%$ respectively.

There were 5 peri-prosthetic fractures. There was one Vancouver type $\mathrm{A}(\mathrm{g})$, two Vancouver Type B2, one Vancouver Type B3 and one Vancouver type C periprosthetic fracture. Vancouver Type A and B2 fractures required ORIF with lateral locking LCDCP only whereas the B3 and Type C required revisions to long stem modular bipolar hemiarthroplasty in addition ${ }^{[15]}$.

Implant breakage was reported in only one patient where in stem broke at its proximal third. Extended trochanteric osteotomy was done and broken implants were retrieved. Long Stem modular bipolar implant was used for subsequent management.

\section{Conclusion}

It can be concluded that uncemented hemiarthroplasty in elderly patients with displaced femoral neck fracture through posterior is associated with a fair number of complications. Intra-operative iatrogenic fractures, mortality during the first postoperative year, dislocation and infection are the most frequent complications. High iatrogenic fracture rate may relate to a longer learning curve with the hemiarthroplasty and a uniform unipolar( Austin Moore) and Bipolar prosthesis stem size for all patients, with most of fractures occurring during stem implantation and reduction of implant head into acetabulum; we recommend using a thorough broach and trial stem in all patients.

A thorough study of pre-operative AP and Lateral X rays and comparisons with opposite side can give an insight into canal diameters and intra-operative need for more articulate broaching and proximal femur bowing where risk of anterior or posterior cortex breach is more.

The dislocation rate co-relates with offset, soft tissue tension and adequate repositioning and closure of short external rotators and capsule. Further large scale studies are required to know if modification in implant choice, surgical techniques and approaches could influence the rate of complications.

\section{References}

1. Cooper C, Campion G, Melton LJ. 3rd. Hip fractures in the elderly: a world-wide projection. Osteoporos Int. 1992; 2:285-289

2. Burge R, Dawson-Hughes B, Solomon DH, Wong JB, King A, Tosteson A. Incidence and economic burden of osteoporosis-related fractures in the United States, 20052025. J Bone Miner Res. 2007; 22:465-475

3. Sadegh Saberi, Aidin Arabzadeh, Bijan Khomeisi, Edris Berehnegard, SM Javad Mortazavi. Early Complications Following Bipolar Hemiarthroplasty for Femoral Neck Fracture in Elderly Patients. Acad J Surg. 2014; 1:3-4

4. Browner BD, Jupiter JB, Levine AM, Trafton PG, Krettek C. Intracapsular Hip Fractures. Skeletal Traum. Ch. 49, 4th Ed. Philadelphia, PA: Saunders Elsevier, 2009.

5. Bucholz R, Court-Brown C, Heckman J, Tornetta P. Rockwood and Green'S Fractures IN Adults. Seventh edition. Wolters Kluwer Lippincott Williams \& Wilkins 2010; 2: 1561-1596.

6. Yusuf Öztürkmen, Mahmut Karamehmetoğlu, Mustafa Caniklioğlu, Yener İnce, and İbrahim Azboy. Cementless hemiarthroplasty for femoral neck fractures in elderly patients. Indian J Orthop. 2008; 42(1):56-60.

7. Shah SN, Wainess RM, Karunakar MA. Hemiarthroplasty for Femoral Neck Fracture in the Elderly: Surgeon and Hospital Volume- Related Outcomes. The J of arthroplasty. 2005; 20(4):503-8.

8. Susanne H, Erik B, Anne G, Johan K, Cecilia R. More hip complications after total hip arthroplasty than after hemiarthroplasty as hip fracture treatment: analysis of 5,815 matched pairs in the Swedish Hip Arthroplasty Register. Acta Orthopaedica. 2020; 91(2):133-138

9. Norrish A, Rao J, Parker MJ. Prosthesis survivorship and clinical outcome of the Austin Moore hemiarthroplasty: an 8-year mean follow-up of a consecutive series of 500 patients. Injury. 2006; 37:734-739.

10. Figved W, Opland V, Frihagen F et al. Cemented versus uncemented hemiarthroplasty for displaced femoral neck fractures. Clin Orthop Relat Res. 2009; 467:2426-2435.

11. Choon Chiet Hong, Nazrul Nashi, Jiong Hao Tan, Ruben 
Manohara, Wei Ting Lee, Diarmuid Paul Murphy. Intraoperative Periprosthetic Femur Fracture during Bipolar Hemiarthroplasty for Displaced Femoral Neck Fractures. Arch Orthop Trauma Surg. 2018; 138(9):11891198.

12. D'Angelo F, Giudici M, Molina M, Margaria G. Mortality rate after hip hemiarthroplasty: analysis of risk factors in 299 consecutives cases. J of Orthop. and Traumatol. 2005; 6(3):111-6

13. Eiskjaer S, Ostgård SE. Risk Factors Influencing Mortality after Bipolar Hemiarthroplasty in the Treatment of Fracture of the Femoral Neck. Clin Orthop Relat Res. 1991; 270:295-300.

14. Chaplin VK, Matharu GS, Knebel RWC. Complications following hemiarthroplasty for displaced intracapsular femoral neck fractures in the absence of routine followup. Ann R Coll Surg Engl 2013; 95:271-274

15. Gaski, Greg E, Sean P Scully. "In brief: classifications in brief: Vancouver classification of postoperative periprosthetic femur fractures." Clinical orthopaedics and related research 2011; 469(5):1507-10.

doi:10.1007/s11999-010-1532-0 